\title{
Familial risk of early and late onset cancer
}

\author{
S Hodgson \\ Professor of Cancer Genetics, St George's, University of London, UK
}

TITLE Familial risk of early and late onset cancer: nationwide prospective study.

AUTHORS Kharazmi E, Fallah M, Sundquist $\mathrm{K}$ et al.

\author{
Correspondence to S Hodgson \\ 40 Onslow Gardens \\ Muswell Hill \\ London NIO 3JU, UK
}

JOURNAL BMJ 2012; 345:e8076.

DECLARATION OF INTERESTS Professor Hodgson is Professor of Cancer Genetics at St George's Hospital and St Georges, University of London. tel. +44 (0)794 6854873

e-mail shodgson@sgul.ac.uk

\section{SUMMARY}

This study, from a very reputable research group, addresses the issue of whether there is a significant familial risk of developing certain common cancers when they develop at a late age (60-76 years old). It is a nationwide prospective study of over 12.2 million individuals in Sweden born after 1931, including over I.I million cases of a first diagnosis of a primary cancer. Data were obtained from the well-established Swedish Cancer Registry, the multigeneration national census register and death registrations, and were linked using individually unique registration numbers. The study evaluated relative risks of the same cancer types in parents and offspring. The cancers were common (colorectal, lung, breast, prostate, urinary, bladder, melanoma, squamous cell carcinoma of the skin and non-Hodgkin's lymphoma). The results showed that the relative risk for developing late onset cancer in individuals with a parent diagnosed with cancer at an elderly age were significant, but were still less than the risks for early onset cancers in offspring with parents with early onset disease.

\section{OPINION}

Previous epidemiological studies focused on the familial risk being more pronounced in early onset cancers; this has left consideration of the familial risk of late onset cancers largely undetermined. Sweden has an excellent family cancer registry database, predicated on the linkage of the National Cancer Registry, multi-generation register, national censuses and death registration, using a unique national registration number, allowing accurate data to be obtained on a very large number of individuals. Data were also available on socioeconomic status, residential area, parental ages for the period of followup, and hospital admissions for obesity, chronic obstructive airways disease and alcohol-related disorders. The hazard ratios were adjusted taking these parameters, as well as age, sex and period of follow-up into account. This allowed the authors to demonstrate that, as found in many previous studies, relatives (offspring) of individuals affected with cancer at young ages had a significantly increased risk of developing the same type of cancer, also at young ages, but that there was also a significantly increased risk (although smaller) for offspring of individuals with late onset cancer (diagnosed at 60-76 years of age) being affected with the same cancer type at late ages. However it appeared that there were no significantly increased risks demonstrated for any of the cancers for offspring diagnosed at later ages whose parents were affected at young ages. Possible problems with the data analysis are discussed in the text, with such issues as 'left truncation of family history' with failure to identify relatives as affected when the disease occurred before the start of registration, or under-ascertainment of risk for elderly patients being addressed, being unlikely to cause serious problems with analysis. There could be a problem with mis-classification of cancers because the study defined concordant cancers as the 'same type of cancer regardless of its histology'. However, for common cancers it is unlikely that this would result in a significant number of discordant cancers being reported erroneously. The table and figure shows that some individuals included in the study were extremely young, but clearly the analysis took this into account. It would have been interesting to see further subdivisions of groups to show the risks in offspring of parents affected with cancer at very early ages ( $<30$ years old), but the study shows that there were high relative risks of early-onset concordant cancer in offspring of parents with young onset cancers of the same type, amounting to hazard ratios of 9.9 in individuals $<40$ years of age with colorectal cancer, for instance, and 5.2 with breast cancer (the most striking risks displayed) but still showing hazard ratios of 1.9 for colorectal cancer and 1.4 for breast cancer in offspring diagnosed at later ages with parents diagnosed at 60-76 years of age. Interestingly, there appeared to be little overlap between the risks conferred by early onset and late onset cancers (except for breast cancer), indicating that the genetic aetiology of cancer susceptibility in these two groups was different.

The authors discuss these outcomes in terms of the importance of recognising the previously unacknowledged familial risks of late-onset cancers, which could emphasise 
the need for preventive lifestyle interventions. There is only limited discussion about the possible different types of gene that could be involved in the two types of susceptibility; early-onset cancers are more likely to be caused by rare germline mutations in strongly or moderately penetrant genes, conferring the very strikingly high hazard ratios observed in young onset cases; late-onset cancers are more likely to be due to a combination of several mutations in common lowpenetrant genes, interacting with environmental factors.
This clearly results in the lower hazard ratios and the authors suggest that such smaller familial risks may be susceptible to lifestyle and environmental modification. It is becoming increasingly appreciated that multiplicative effects of low-penetrance genetic variants may explain the 'missing heritability of cancers', and it seems highly plausible that these might confer increased risks of late onset rather than early onset cancers. This paper provides interesting epidemiological evidence in accordance with this theory.

\section{FURTHER READING}

I Dunlop MG, Tenesa A, Farrington SM et al. Cumulative impact of common genetic variants and other risk factors on colorectal cancer risk in 42103 individuals. Gut 2012; Epub ahead of print. http://dx.doi.org/I0.I I36/gutjnl-20 I I-300537

2 Houlston RS, members of COGENT. COGENT (Colorectal cancer GENeTics) revisited. Mutagenesis 20I2: 27:I43-5I. http:// dx.doi.org// 0.1093/mutage/ger059

3 Tomlinson IP, Carvajal-Carmona LG, Dobbins SE et al. Multiple common susceptibility variants near BMP pathway loci GREMI, BMP4, and BMP2 explain part of the missing heritability of colorectal cancer. PLoS Genet 20II; 7:el002105. http://dx.doi. org/10.137I/journal.pgen.1002105

4 Niittymäki I, Kaasinen E, Tuupanen S et al. Low-penetrance susceptibility variants in familial colorectal cancer. Cancer Epidemiol Biomarkers Prev 2010 19:1478-83. http://dx.doi.org/I0.II58/I0559965.EPI-09-1320

5 Aaltonen L, Johns L, Järvinen $\mathrm{H}$ et al. Explaining the familial colorectal cancer risk associated with mismatch repair (MMR)deficient and MMR-stable tumors. Clin Cancer Res 2007; I 3:356-6I. http://dx.doi.org/I0.II58/I078-0432.CCR-06-1256

Royal College of Physicians of Edinburgh

\section{Writing a journal article}

Have you ever had a paper rejected for publication?

If so, have you ever wondered why it was rejected?

Would you like to improve your publication rate or the level of publication in which your papers appear?

The Writing a journal article course aims to assist RCPE Fellows, Collegiate Members and Associates, and their colleagues, in improving their publication rates through gaining a better understanding of what journals look for in submitted papers.

This course is designed for people who are at, or nearly at, the stage of putting together a paper for publication.

Writing a journal article provides valuable insights into how to plan, structure, develop and write a scientific paper; information on what editors and reviewers look for in papers; and original analyses of trends in medical publishing.

Next courses: Monday 4 March and Monday 7 October 2013

More information will be available in January on the

College website http://events.rcpe.ac.uk/

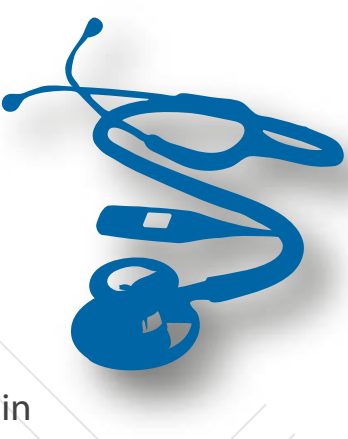

